\title{
Repair Integrity and Functional Outcomes after Arthroscopic Repair of Transtendinous Full-thickness Rotator Cuff Tears Minimum Two-year Follow-up
}

\author{
Kyung Cheon Kim, Woo-Yong Lee ${ }^{1 凶}$, Hyun Dae Shin ${ }^{1}$, Young-Mo Kim ${ }^{1}$, Sun Cheol Han \\ Shoulder Center and Department of Orthopedic Surgery, TanTan Hospital, 'Department of Orthopedic Surgery, Chungnam National University School of Medicine, \\ Daejeon, Korea
}

\begin{abstract}
Background: To evaluate the clinical outcomes and associated repair integrity in patients treated with arthroscopic repair for a transtendinous rotator cuff tear followed by resection of the remnant rotator cuff tendon.

Methods: Between July 2007 and July 2011, we retrospectively reviewed patients who were treated for transtendinous full-thickness tears in the tendinous portion of the rotator cuff by arthroscopic repair. Clinical outcomes were evaluated using the American Shoulder and Elbow Surgeons (ASES) score, the Shoulder Rating Scale of the University of California at Los Angeles (UCLA), the Constant-Murley score, a visual analogue scale (VAS) pain score, and range of motion (ROM). The repair integrity was determined by magnetic resonance imaging or ultrasonography.

Results: There were 19 shoulders with transtendinous full-thickness tears in the tendinous portion of the rotator cuff. The ASES, UCLA, Constant-Murley, and VAS pain scores showed significant improvements in function and symptoms (all $p<0.001$ ). The active ROM for forward flexion and abduction was also significantly improved ( $p=0.002$ and $p<0.001$, respectively). The postoperative radiological examination showed cuff integrity without a re-tear in $68.4 \%$ of patients. However, the UCLA, ASES, and Constant-Murley scores were not significantly different between healed and re-torn group $(p=0.530, p=0.885$, and $p=0.262$, respectively).

Conclusions: Although repair of transtendinous rotator cuff tears followed by resection of the remnant rotator cuff tendon in the footprint has a relatively high re-tear rate, no significant difference was observed in the short-term clinical results between the re-tear and healed groups.
\end{abstract}

(Clin Shoulder Elbow 2017;20(4):183-188)

Key Words: Rotator cuff; Transtendinous tear; Arthroscopy

\section{Introduction}

Full-thickness rotator cuff tears transversely involving the musculotendinous portion without tear extension to the footprint of the greater tuberosity are very rarely observed during arthroscopic operation, and few studies have investigated this tear pattern. Walcott et al. ${ }^{1)}$ defined this tear pattern as a transtendinous rotator cuff tear. Full-thickness defects in the musculotendinous area with the remaining rotator cuff on the greater tuberosity may be revealed on oblique coronal views or long-axis views upon magnetic resonance imaging (MRI). The torn cuffs of such tears is difficult to repair because of a shortage of references. It is also difficult for surgeons to determine whether it is better to repair the torn cuff to the remnant rotator cuff on the footprint or repair the torn cuff to the footprint after resection of the remnant cuff on the footprint to enhance the healing potential of the repaired tendon. ${ }^{1)}$

To the best of our knowledge, no studies conducted to date

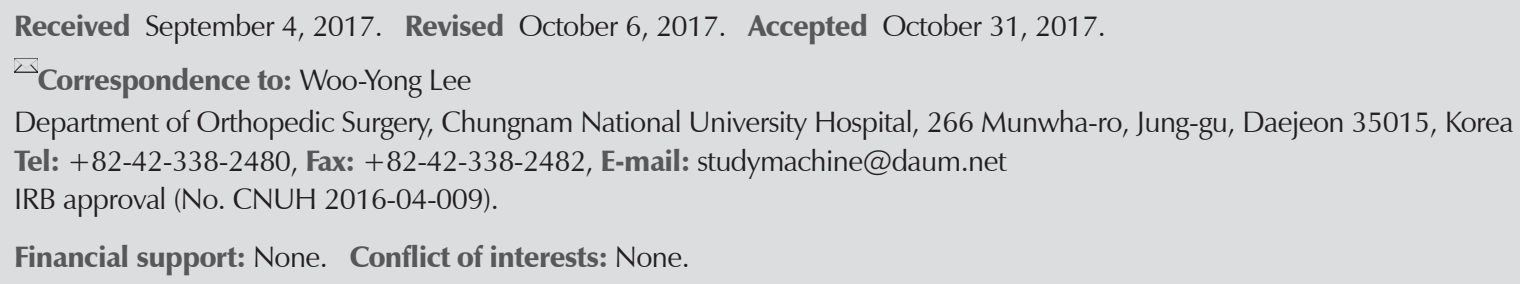


have included a larger number of cases of transtendinous rotator cuff tears than ours. In addition, transtendinous rotator cuff repair integrity after arthroscopic repair for transtendinous rotator cuff tears has not been reported. Therefore, the present study was conducted to evaluate the clinical outcomes and associated repair integrity in patients treated with arthroscopic repair for a transtendinous rotator cuff tear followed by resection of the remnant rotator cuff tendon. Our hypothesis was that the clinical results would be improved in patients treated for transtendinous full-thickness rotator cuff tears after arthroscopic repair.

\section{Methods}

\section{Patient Selection}

Between July 2007 and July 2011, we retrospectively reviewed patients who were treated for transtendinous fullthickness rotator cuff tears by arthroscopic repair. We defined the transtendinous full-thickness rotator cuff tears as those within the tendinous portion of the rotator cuff, with most of the tendon footprint intact and at least $1 \mathrm{~cm}$ of attached tendon as Walcott et al. ${ }^{1)}$ reported. Consequently, we included patients with transtendinous full-thickness rotator cuff tears when the tear was observed in the tendinous or musculotendinous area during arthroscopic examination (Fig. 1). However, oblique transtendinous rotator cuff tears that were extended to the footprint on arthroscopic views (similar to L-shaped or reverse L-shaped tears) were excluded from this study. In addition, we excluded patients with the following: (i) rotator cuff tears that could be reduced by the free tendon edge to the footprint, such as oblique L-shaped or reverse L-shaped tears (oblique transverse tears) that could be repaired side-to-side after fixing the tendon edge to the footprint, (ii) full-thickness subscapularis tears requiring repair, (iii) revision operations, (iv) neurological involvement, (v) arthritic changes in the glenohumeral joint on radiographs, and (vi) incomplete hospital record including postoperative clinical and radiological evaluation.

\section{Surgical Technique and Evaluation}

All operations were performed by a single surgeon (first author) with the patient under general anesthesia and in the beach-chair position. Diagnostic arthroscopy was performed to assess the rotator cuff and associated pathology. Subacromial decompression was performed in patients with evidence of subacromial impingement and confirmed by arthroscopy.

After removal of the remaining rotator cuff on the footprint and release of adhesions and mobilization of the tendon, the surgeon attempted to repair the free tendon edge on the footprint of the greater tuberosity. Suture anchors were employed to repair the rotator cuff on the footprint using several fixation techniques depending on tissue quality and mobility. When
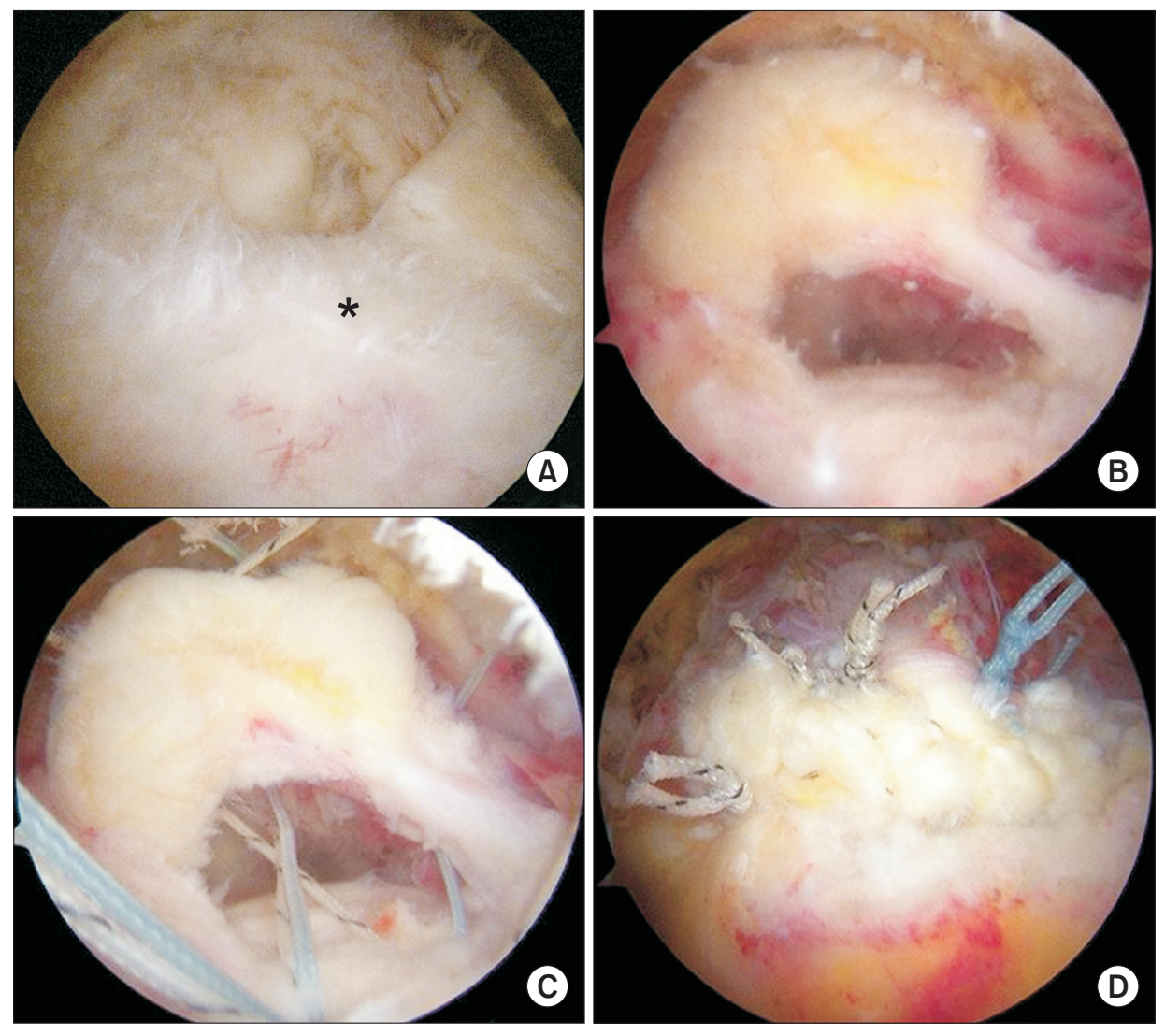

Fig. 1. The arthroscopic view showed the remnant (asterisk) of the rotator cuff on the footprint (A) and status after removal of the remnant on the footprint, release of adhesions and mobilization of the tendon (B). (C, D) After attempting to repair the free tendon edge on the footprint, suture anchors were placed to repair the rotator cuff on the footprint using the single-row technique. 
direct repair of the rotator cuff tear was impossible, as with longitudinal-type tears exhibiting a long and narrow pattern with an increased medial-to-lateral length after resection of the remnant rotator cuff on the footprint, medialization or margin convergence of the torn rotator cuff was attempted. The rotator cuff was repaired using a single-row technique alone (five shoulders), single-row technique after margin convergence (eight shoulders), double-row technique (one shoulder), or suture-bridge technique (six shoulders). The inclusion criteria for biceps tenotomy were a rotator cuff tear combined with a biceps lesion, such as a tear of more than $30 \%$ of the normal width of the tendon, subluxation or dislocation, a degenerative superior labrum anterior to posterior (SLAP) type II lesion, and age greater than 55 years.

The maximum anterior-to-posterior (AP) and medial-tolateral $(\mathrm{ML})$ length of each rotator cuff tear and the $\mathrm{ML}$ length of the remnant rotator cuff on the footprint was measured using a calibrated probe under arthroscopic observation after removal of the remaining cuff on the footprint. All measurements were made by the first author (Table 1).

\section{Functional Evaluations}

Five outcome measures were used: the American Shoulder and Elbow Surgeons (ASES) score, ${ }^{2)}$ the Shoulder Rating Scale of the University of California at Los Angeles (UCLA), ${ }^{3)}$ the Constant-Murley score, ${ }^{4)}$ a visual analogue scale (VAS) pain score, and range of motion (ROM). Pain was recorded using a VAS. Active ROM was measured by goniometry, while passive ROM was not measured.

\section{Radiological Evaluations}

Rotator cuff repair integrity was determined by MRI or ultra-

Table 1. Demographic and Surgical Data

\begin{tabular}{|cc|}
\hline \multicolumn{1}{|c}{ Variable } & Value \\
\hline No. of patient & 19 \\
\hline Patients' characteristic & \\
\hline Age at surgery (yr) & $61.0(44-72)$ \\
\hline Sex (male) & 8 \\
\hline Affected shoulder (right) & 10 \\
\hline Duration of symptoms $(\mathrm{mo})$ & $9.1(1-60)$ \\
\hline Smoking & 2 \\
\hline Tear size (cm) & $2.1(1.2-3.5)$ \\
\hline AP dimension & $2.5(1.5-3.6)$ \\
\hline ML dimension & \\
\hline Remaining rotator cuff on footprint $(\mathrm{cm})$ & $1.6(1.0-2.2)$ \\
\hline ML dimension &
\end{tabular}

Values are presented as number only or median (range). AP: anterior-to-posterior, ML: medial-to-lateral. sonography (US) evaluation at least 6 months after arthroscopic repair for rotator cuff tears. We performed US evaluation in patients who refused MRI evaluation for economic reasons or claustrophobia during the MRI examination. The MRI and US findings were evaluated by an experienced radiologist. In particular, one musculoskeletal radiologist conducted all follow-up US examinations using an IU-22 system (Philips Healthcare, Bothell, WA, USA). The surgeon informed the radiologist about the surgical procedure and postoperative configuration before evaluation of the cuff integrity. A recurrent tendon defect was diagnosed with US when a distinct hypoechoic or mixed hyperechoic and hypoechoic defect was visualized in both the transverse and longitudinal planes. A full-thickness re-tear was diagnosed if there was a focal defect in the rotator cuff into which the deltoid muscle could be compressed with a probe to separate the torn tendon ends or if the cuff retracted to such an extent that the torn ends could be distinguished. The integrity of the tendon was classified into two categories using MRI: (1) intact (sufficient thickness) or (2) insufficient/not healed/re-torn (from insufficient thickness with less than half the thickness of a normal cuff up to discontinuity). ${ }^{5)}$

\section{Statistical Analysis and Research Approval}

Paired t-tests were used to assess differences in preoperative and postoperative results. IBM SPSS ver. 20.0 (IBM Co., Armonk, NY, USA) was used for all statistical analyses, with an $\alpha$ level of 0.05 taken to indicate significance. The Mann-Whitney U-test was used to compare the re-tear and no re-tear groups. This study was approved by Institutional Review Board of Chungnam National University Hospital and the need for informed consent from all patients was waived.

\section{Results}

\section{Arthroscopic Findings}

Overall, 19 consecutive shoulders (8 males, 11 females) who met the inclusion criteria (and not any exclusion criterion) were identified for a total of ten affected right and nine affected left shoulders (Table 1). The mean tear size was $2.1 \pm 0.17 \mathrm{~cm}$ (range, 1.2-3.5 cm) in the AP dimension and $2.5 \pm 0.15 \mathrm{~cm}$ (range, 1.5-3.6 cm) in the $\mathrm{ML}$ dimension. The mean size of the remnant rotator cuff on the footprint was $1.6 \pm 0.07 \mathrm{~cm}$ (range, $1.0-2.2 \mathrm{~cm}$ ) in the ML dimension (Table 1). Six patients had associated long head biceps (LHB) lesions, including four patients with a partial tear. Associated surgical procedures (LHB tenotomy) were performed at the time of cuff repair in these patients. Arthroscopic subacromial decompression was performed in all patients.

\section{Functional Results}

The mean time from surgery to the postoperative final follow- 
up evaluation was 29.3 months (range, 24-50 months). The mean ASES score was improved from $46.0 \pm 9.6$ (range, 30-60) preoperatively to $91.3 \pm 14.6$ (range, 41.6-100) at the last follow-up. The mean UCLA score was improved from $19.6 \pm$ 3.9 (range, 14-27) preoperatively to $33.0 \pm 3.3$ (range, 21-35) at the last follow-up. The mean Constant-Murley score was improved from $52.1 \pm 12.6$ (range, 28-68) preoperatively to $81.0 \pm 14.6$ (range, 50-100) at the last follow-up. All functional scores showed significant improvements (all $p<0.001$ ). The mean VAS was improved from $5.6 \pm 1.0$ (range, 4-7) to $1.1 \pm$ 2.1 (range, 0-9) $(p<0.001)$. The mean active ROM for forward flexion was improved from $142.1^{\circ} \pm 41.6^{\circ}$ (range, 80 $-180^{\circ}$ ) preoperatively to $175.8^{\circ} \pm 6.1^{\circ}\left(\right.$ range, $\left.160^{\circ}-180^{\circ}\right)(p=0.002)$ at the last follow-up; abduction was improved from $120.5^{\circ} \pm$ $43.5^{\circ}$ (range, $20^{\circ}-180^{\circ}$ ) to $176.8^{\circ} \pm 9.5^{\circ}$ (range, $150^{\circ}-180^{\circ}$ ) $(p<0.001)$.

\section{Radiological Results}

Nineteen patients underwent postoperative MRI $(n=6)$ or US $(n=13)$ examination after the operation. The mean final followup period for the radiological evaluation was 14.2 months (range, 6-34 months). The postoperative radiological evaluation for repair integrity showed a re-tear in six shoulders (31.6\%), including five shoulders with a partial-thickness re-tear and one shoulder with a full-thickness re-tear (Fig. 2). Four shoulders with a partialthickness re-tear were identified by US, while two shoulders with a partial-thickness and a full-thickness re-tear on the footprint were identified by MRI. Four partial-thickness re-tears were revealed in the musculotendinous junction, and one articular side partial-thickness re-tear and one full-thickness re-tear in the footprint were revealed. However, re-tears tended to be smaller than the original tear. In addition, the UCLA, ASES, and Constant-Murley scores were not significantly different between the healed and re-torn group after repairing transtendinous rotator cuff tears ( $p=0.530, p=0.885, p=0.262$, respectively) (Table
2). No clinical failures were observed for symptomatic re-tears in patients who underwent an arthroscopic revision operation. No nerve damage, deep infection, or anchor-related complications occurred in any patients.

\section{Discussion}

Our results showed that the re-tear rate was $31.6 \%$ in patients treated for transtendinous full-thickness rotator cuff tears after arthroscopic repair, despite the improved short-term clinical results. Although many other factors can affect outcomes, the clinical outcomes were found to improve regardless of tendon integrity. However, re-tears tended to be smaller than the original tear.

In a study on medial rotator cuff failure after arthroscopic double-row rotator cuff repair, a likely cause of medial cuff failure was found to be tension overload of the suture-tendon interface at the medial row, which becomes the tension-bearing

Table 2. Comparison of the Preoperative State and Postoperative Clinical Outcomes of the Final Follow-up

\begin{tabular}{lrcc}
\multicolumn{1}{c}{ Outcome } & Healed patient & Re-tear patient & $p$-value \\
\hline VAS & $5.6 \pm 1.0$ & $1.1 \pm 2.1$ & $<0.001$ \\
ASES & $46.0 \pm 9.6$ & $91.3 \pm 14.6$ & $<0.001$ \\
UCLA & $19.6 \pm 3.9$ & $33.0 \pm 3.3$ & $<0.001$ \\
Constant-Murley score & $52.1 \pm 12.6$ & $81.0 \pm 14.6$ & $<0.001$ \\
ROM $\left(^{\circ}\right)$ & & & \\
\hline Forward flexion & $142.1 \pm 41.6$ & $175.8 \pm 6.1$ & 0.002 \\
\hline Abduction & $120.5 \pm 43.5$ & $176.8 \pm 9.5$ & $<0.001$ \\
\hline
\end{tabular}

Values are presented as mean \pm standard deviation.

VAS: visual analogue scale pain score, ASES: American Shoulder and Elbow Surgeons score, UCLA: Shoulder Rating Scale of the University of California at Los Angeles, ROM: range of motion.

${ }^{*}$ Based on separate paired t-test; $p<0.05$ denotes statistical significance.
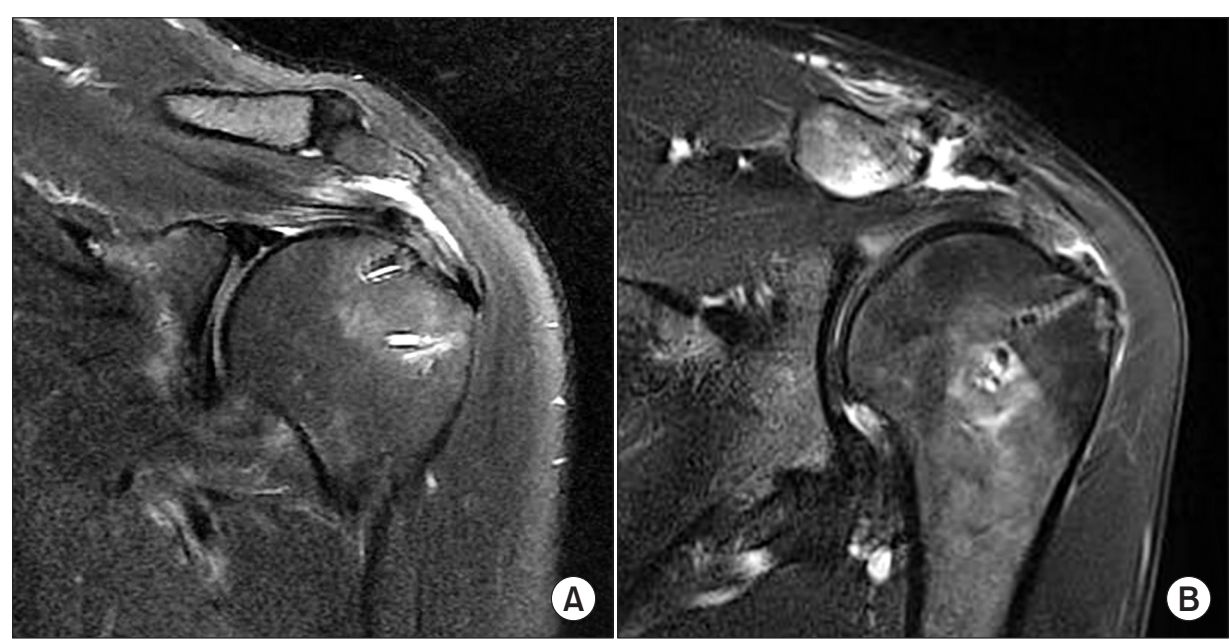

Fig. 2. Postoperative radiological evaluation of repair integrity showed a well-healed rotator cuff (A) and full-thickness re-tear around the footprint (B). 
row. ${ }^{6)}$ In addition, medial rotator cuff failure mainly at the musculotendinous junction after arthroscopic suture-bridge rotator repair tends to better preserve the cuff tissue repaired to the insertion site of the rotator cuff than the single-row technique. ${ }^{\text {) }}$ We thought that one possible pathogenesis of this type of tear was an insufficient supply or ischemic condition of unknown origin in the musculotendinous area of the rotator cuff. Although the pathogenesis of transtendinous rotator cuff tears was not revealed in this study, it may be clinically important for surgeons to choose a repair method that enhances the healing potential of the repaired tendon between a remnant-preserving and remnant-sacrificing method. However, neither a detailed description nor repair integrity for primary transtendinous rotator cuff tears has been reported. Indeed, only one Walcott et al. ${ }^{1)}$ reported the clinical outcomes of transtendinous rotator cuff repairs following their repair. Seven patients underwent arthroscopic or open surgery and torn cuffs were remnant-preserving repaired in a side-to-side fashion using one limb of suture through the lateral tendon and one limb through the medial tendon. Although their clinical outcomes were good, radiological outcome was not evaluated.

On the free tendon edge of some U-shaped, full-thickness rotator cuff tears that cannot be reduced to the footprint, the margin convergence technique (side-to-side tendon repair) may be indicated. This margin convergence has a significant re-tear rate $(47.3 \%)^{8)}$ In transtendinous full-thickness rotator cuff tears, proximal-to-distal tendon repair without sacrifice of the remaining cuff on the footprint may be much more vulnerable to postoperative re-tear than side-to-side tendon repair, although Walcott et al." ${ }^{1)}$ claimed the remnant-sacrificing method would over-tension the repair, jeopardizing tendon healing. In addition, the remnant-preserving method in which the proximal torn tendon is directly repaired to the distal remaining rotator cuff on the footprint using few suture materials may be expected to have a high re-tear rate of the repaired cuff and weak mechanical strength for repair site maintenance. It was also technically difficult to pass a sufficient number of suture materials between the proximal and distal torn tendons for repair.

In this study, we performed a complete removal of the remnant cuff on the footprint and directly repaired the torn cuff on the decorticated footprint using suture anchors to enhance the healing potential and repair the footprint. However, this remnant-sacrificing method also has some potential limitations. First, direct reduction of the torn cuff to the footprint may be difficult because of the loss of tendon length, and the resultant undue tension is not desirable for cuff healing. ${ }^{9)}$ Therefore, when direct repair of a rotator cuff tear on the footprint is impossible, medialization or margin convergence of the torn rotator cuff is needed. Second, avoiding passage of sutures through the medial portion of the tendon may be beneficial for reducing the re-tear rates. However, attempts to achieve a more medial suture tendon passage owing to a reduced tendon length have led to tendon passage at the musculotendinous junction instead of the tendon portion, making the tendons vulnerable to re-tearing. ${ }^{6,7)}$ In our study, $66.7 \%(4 / 6)$ of re-tears occurred in the musculotendinous area.

Although the postoperative functional scores were not significantly different between the healed and re-torn group in our study, this does not mean that the postoperative repair integrity was not important or not correlated with clinical improvement. For rotator cuff tears, the integrity of the repair site after surgery is correlated with clinical improvement, particularly the return of strength. ${ }^{10-12)}$ However, re-tears after arthroscopic rotator cuff repair may occur for various reasons, including poor-quality tendon tissue, loss of suture anchors, suture breakage, and inappropriate rehabilitation. ${ }^{13-20)}$ Many studies have shown that pain and overall scores are comparable between re-tear and non-retear cohorts, but that if sufficiently powered with subscales of strength and function broken out, intact repairs have better outcomes. Our study included too few patients and did not report strength data; therefore, we were unable to address this issue. In addition, Yamaguchi et al. ${ }^{21)}$ found tear extension in 39\% of patients in a longitudinal study using US for nonhealing rotator cuff tendons. This is especially important given the evidence that fullthickness rotator cuff tears do not heal spontaneously. It has long been noted that rotator cuff tears retract and form adhesions, complicating surgical repair. Tendons deteriorate over time, often leaving only tissue-paper-quality tendons for repair. Fatty degeneration and muscle atrophy occur and are likely permanent changes in longstanding cuff tears.

It should be noted that our study had some limitations. First, there was no control group with which to directly compare the remnant-sacrificing method with another remnant-preserving repair method. However, our study is the largest therapeutic case series, although transtendinous tear is rare. Second, although all patients were evaluated using US by an experienced musculoskeletal radiologist, this method is examiner-dependent. However, we did not perform the US examination ourselves to avoid prejudice. Third, we could not compare the postoperative cuff integrity evaluation by the two radiologists to compare the reliability of US and MRI. Fourth, we were unable to assess the preoperative muscle atrophy grades because of incomplete MRI scans without an appropriate Y-shaped view. Fifth, the number of shoulders included was too few to generate clinically significant results. Finally, the repair techniques were not homogenous.

\section{Conclusion}

Although repair of transtendinous rotator cuff tears followed by resection of the remnant rotator cuff tendon in the footprint has a relatively high re-tear rate, no significant difference was observed in the short-term clinical results between the re-tear 
and healed groups. We believe our study will be good reference for evaluation of transtendinous full-thickness rotator cuff tear.

\section{References}

1. Walcott ME, Daniels SD, Sinz NJ, Field LD, Higgins LD. Traumatic full-thickness transtendinous rotator cuff tears: a case series. J Shoulder Elbow Surg. 2017;26(1):62-7.

2. Richards RR, An KN, Bigliani LU, et al. A standardized method for the assessment of shoulder function. J Shoulder Elbow Surg. 1994;3(6):347-52.

3. Ellman H, Hanker G, Bayer M. Repair of the rotator cuff. Endresult study of factors influencing reconstruction. J Bone Joint Surg Am. 1986;68(8):1136-44.

4. Constant CR, Murley AH. A clinical method of functional assessment of the shoulder. Clin Orthop Relat Res. 1987;(214): 160-4.

5. Sugaya H, Maeda K, Matsuki K, Moriishi J. Functional and structural outcome after arthroscopic full-thickness rotator cuff repair: single-row versus dual-row fixation. Arthroscopy. 2005;21(11):1307-16.

6. Trantalis JN, Boorman RS, Pletsch K, Lo IK. Medial rotator cuff failure after arthroscopic double-row rotator cuff repair. Arthroscopy. 2008;24(6):727-31.

7. Cho NS, Yi JW, Lee BG, Rhee YG. Retear patterns after arthroscopic rotator cuff repair: single-row versus suture bridge technique. Am J Sports Med. 2010;38(4):664-71.

8. Kim KC, Shin HD, Cha SM, Kim JH. Repair integrity and functional outcomes for arthroscopic margin convergence of rotator cuff tears. J Bone Joint Surg Am. 2013;95(6):536-41.

9. Meyer DC, Wieser K, Farshad M, Gerber C. Retraction of supraspinatus muscle and tendon as predictors of success of rotator cuff repair. Am J Sports Med. 2012;40(10):2242-7.

10. Bishop J, Klepps S, Lo IK, Bird J, Gladstone JN, Flatow EL. Cuff integrity after arthroscopic versus open rotator cuff repair: a prospective study. J Shoulder Elbow Surg. 2006;15(3):290-9.

11. Jost B, Pfirmann CW, Gerber C, Switzerland Z. Clinical out- come after structural failure of rotator cuff repairs. J Bone Joint Surg Am. 2000;82(3):304-14.

12. Liu SH, Baker CL. Arthroscopically assisted rotator cuff repair: correlation of functional results with integrity of the cuff. Arthroscopy. 1994;10(1):54-60.

13. Barber FA, Coons DA, Ruiz-Suarez M. Cyclic load testing of biodegradable suture anchors containing 2 high-strength sutures. Arthroscopy. 2007;23(4):355-60.

14. Bardana DD, Burks RT, West JR, Greis PE. The effect of suture anchor design and orientation on suture abrasion: an in vitro study. Arthroscopy. 2003;19(3):274-81.

15. Bynum CK, Lee S, Mahar A, Tasto J, Pedowitz R. Failure mode of suture anchors as a function of insertion depth. Am J Sports Med. 2005;33(7):1030-4.

16. Cummins CA, Murrell GA. Mode of failure for rotator cuff repair with suture anchors identified at revision surgery. J Shoulder Elbow Surg. 2003;12(2):128-33.

17. Gerber C, Meyer DC, Frey E, et al. Neer Award 2007: reversion of structural muscle changes caused by chronic rotator cuff tears using continuous musculotendinous traction. An experimental study in sheep. J Shoulder Elbow Surg. 2009;18(2): 163-71.

18. Goutallier D, Postel JM, Gleyze P, Leguilloux P, Van Driessche S. Influence of cuff muscle fatty degeneration on anatomic and functional outcomes after simple suture of full-thickness tears. J Shoulder Elbow Surg. 2003;12(6):550-4.

19. Mahar A, Allred DW, Wedemeyer M, Abbi G, Pedowitz R. A biomechanical and radiographic analysis of standard and intracortical suture anchors for arthroscopic rotator cuff repair. Arthroscopy. 2006;22(2):130-5.

20. Su WR, Budoff JE, Luo ZP. The effect of anterosuperior rotator cuff tears on glenohumeral translation. Arthroscopy. 2009; 25(3):282-9.

21. Yamaguchi K, Tetro AM, Blam O, Evanoff BA, Teefey SA, Middleton WD. Natural history of asymptomatic rotator cuff tears: a longitudinal analysis of asymptomatic tears detected sonographically. J Shoulder Elbow Surg. 2001;10(3):199-203. 